\title{
miR-184 Inhibits Tumor Invasion, Migration and Metastasis in Nasopharyngeal Carcinoma by Targeting Notch2
}

\author{
Hong-Ming Zhu ${ }^{a} \quad$ Xue-Song Jiang ${ }^{a, b}$ Hui-Zi Lib Lu-Xi Qian ${ }^{a, b}$ \\ Ming-Yu Du ${ }^{a, c} \quad Z$ hi-Wei Lu, Jing Wu ${ }^{a}$ Xiao-Kang Tian ${ }^{a, c}$ \\ Qian Fej ${ }^{a, b} \quad$ Xia He $\mathrm{H}^{\mathrm{a}, \mathrm{b}, \mathrm{c}}$ Li Yin ${ }^{\mathrm{a}, \mathrm{b}}$
}

ajiangsu Cancer Hospital, Jiangsu Institue of Cancer Research, Nanjing Medical University Affiliated

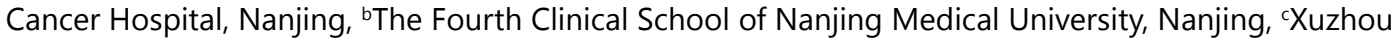
Medical University, Xuzhou, China

\section{Key Words}

miR-184 • Nasopharyngeal carcinoma • Notch2 • Invasion • Metastasis • EMT

\begin{abstract}
Background/Aims: A recent study found that dysregulated microRNA-184 (miR-184) is involved in the proliferation and survival of nasopharyngeal carcinoma (NPC). This study aimed to evaluate the detailed mechanisms of invasion, migration and metastasis of NPC cells. Methods: Quantitative reverse-transcription PCR (qRT-PCR) and Western blot were used to confirm the expression levels of miR-184 and Notch2. NPC cell invasion and migration were subsequently examined using in vitro cell invasion and wound-healing assays, respectively. MicroRNA (miRNA) target gene prediction databases and dual-luciferase reporter assay were adopted to validate the target genes of miR-184. Results: MiR-184 was downregulated in the NPC cell lines. The miR-184 inhibitor increased the number of invading NPC cells, whereas miR-184 mimics inhibited the invasive ability of such cells. The protein level of E-cadherin decreased, whereas those of $\mathrm{N}$-cadherin and vimentin increased in the anti-miR-184 group. This result showed that miR-184 inhibited NPC cell invasion and metastasis by regulating EMT progression. MiRNA target gene prediction databases indicated the potential of Notch2 as a direct target gene of miR-184. Such a notion was then validated by results of dual-luciferase reporter assay. Notably, shRNANotch2 restrained the EMT and partially abrogated the inhibitory effects of miR-184 on EMT progression in NPC cells. Conclusion: MiR-184 functions as a tumour-suppressive miRNA targeting Notch2 and inhibits the invasion, migration and metastasis of NPC.

(C) 2018 The Author(s)

Published by S. Karger AG, Basel

H.-M. Zhu and X.-S. Jiang contributed equally to this work.

\begin{tabular}{ll}
\hline Xia He & Jiangsu Cancer Hospital, Jiangsu Institue of Cancer Research, Nanjing Medical University \\
and Li Yin & Affiliated Cancer Hospital, 42 Bai Zi Ting Road, Nanjing, Jiangsu 210000 (China) \\
& E-Mail hexiabm@163.com; yinli_2012@126.com
\end{tabular}
\end{abstract}




\section{Introduction}

Nasopharyngeal carcinoma (NPC) is a common head and neck malignant tumour in the people of South China, particularly in Guangdong Province, Guangxi Province, Hunan Province, Fujian Province, Jiangxi Province, and Hong Kong Special Administrative Region [1]. Radiotherapy is a commonly used method for treating NPC and is combined with chemotherapy to improve patient survival rate [2]. However, NPC metastasis is an important cause of poor survival among patients with advanced NPC[3]. The underlying molecular mechanisms in NPC metastasis remain unclear. Therefore, the molecular mechanisms of NPC invasion and metastasis must be urgently elucidated to improve the prognosis of patients with NPC.

MicroRNAs (miRNAs) are a class of endogenous shortnon-codingRNAs, which can repress gene expression by base pairing to specific recognition sequences in the $3^{\prime}$-untranslated region (UTR) of their target mRNAs [4]. Accumulating evidence has shown that miRNAs are associated with tumourigenesis, progression and metastasis either as oncogenes or tumour suppressors in NPC and other cancers by regulating target gene expression [5-7]. Several miRNAs have been reported to serve as oncogenes or suppressor genes in NPC cell migration, invasion and metastasis. These miRNAs include miR-145 [8], miR-429 [9], miR-143 [10] and miR-148-b [11]. These studies suggest that miRNAs play important roles in NPC and provide a novel area of study in NPC invasion and metastasis.

MiRNA-184 (miR-184) is an evolutionarily conserved non-coding RNA oligomer located in region 25.1 of the q-arm of chromosome 15. Mature miR-184, which contains 22 nucleotides, exhibits expression patterns specific to tissue and developmental stages [12]. It has been extensively reported that miR-184 activity participates in the control of a wide range of biological functions and processes such as changes in response to DOR activation in the Rat Liver Under Prolonged Hypoxia [13]. MiR-184 has been extensively explored in various cancers. Decreased miR-184 expression levels were noted in gliomal cells [14, 15], central nervous system lymphoma cells [16] and renal cell carcinoma [17]. By contrast, miR-184 expression was found to be upregulated in pancreatic ductal adenocarcinoma [18], hepatocellular carcinoma [19] and tongue squamous cell carcinoma [20]. In a previous study, dysregulated miR-184 contributed to growth and apoptosis [21]. However, the role of miR184 in NPC invasion and metastasis remains unclear; thus, it was investigated in this study.

\section{Materials and Methods}

\section{Cell lines and Cell culture}

Six NPC cell lines (5-8F, 6-10B, HNE1, SUNE1, CNE-1, and CNE-2) were cultured in RPMI-1640 (Hyclone, Logan, UT, USA ) and immortalized nasopharyngeal epithelial cell NP69 supplemented with 5\% FBS (Gibco BRL, Gaithersburg, MD, USA) in a humidified atmosphere of $5 \% \mathrm{CO} 2$ at $37^{\circ} \mathrm{C}$.

\section{Cell transfection}

hsa-miR-184 mimic/inhibitor and hsa-miR-184 mimic/inhibitor negative control (NC) were synthesized by RiboBio (Guangzhou, China). Notch2-siRNA and NC siRNA were obtained from RiboBio (Guangzhou, China). CNE- 1 and CNE- 2 cells were plated in six-well plates $\left(1.5 \times 10^{5}\right.$ cells per well) for $24 \mathrm{~h}$ and transfected with miR-184 mimic/inhibitor/Notch2-siRNA using Lipofectamine 2000 (Invitrogen, Carlsbad, $\mathrm{CA}$ ) according to the manufacturer's protocol. Cells transfected with miRNA or siRNA were harvested $48 \mathrm{~h}$ post-transfection.

\section{Quantitative real-time PCR assays}

Total RNA was purified using TRIzol reagent (Invitrogen,Carlsbad, CA) according to the manufacturer's instructions to detect mRNA expression. Then, $2 \mu \mathrm{g}$ of total RNA was reverse-transcribed using Moloney murine leukemia virus reverse transcriptase (Promega, Madison, WI, USA) according to manufacturer's instructions. qRT-PCR was performed using an ABI 7300 FAST Real-Time PCR System (Applied Biosystems, Carlsbad, CA, USA) and a SYBR Green PCR Kit (Takara, Dalian,China). The relative expression level of miRNA or mRNA was quantified with the $2^{-\Delta \Delta c t}$ method after normalization with the endogenous reference U6 


\section{Cellular Physiology Cell Physiol Biochem 2018;49:1564-1576

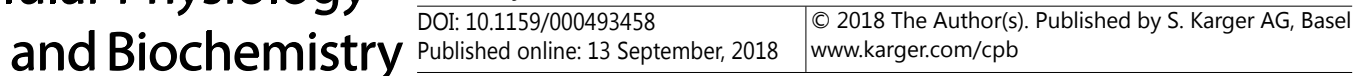 \\ Zhu et al.: miR-184 Inhibits Tumor Invasion}

small nuclear RNA or $\beta$-actin , respectively. The expression of Notch 2 and $\beta$-actin was examined using the following specific primers: 5'-CAAGGAACCTGCTTTGATGACA-3'and 5'-GGGGAACAGGGAGCCAATAC-3 and 5'-GGACTTCGAGCAAGAGATGG-3' and 5'-AGCACTGTGTTGGCGTACAG-3', respectively. All reactions were performed in triplicate for each sample. Fold changes for miR-184 and Notch2 expression levels were calculated using the $2^{-\Delta \Delta \mathrm{Ct}}$ method.

\section{Transwell invasion and migration assay}

Cell invasion was conducted using Matrigel invasion assay (BD Biosciences) according to the manufacturer's protocols. Transfected CNE-1 and CNE-2 cells were cultured for $48 \mathrm{~h}$. The cells were then harvested, resuspended ( $5 \times 104$ cells per well) in $200 \mu$ l of serum-free media, and placed in the upper compartment of a chamber (Corning, NY, USA). The lower compartment was filled with 500 $\mu$ l of RPMI-1640 and $20 \%$ FBS. After $24 \mathrm{~h}$ of incubation, cells that invaded through the Matrigel membrane were fixed with $4 \%$ paraformaldehyde and stained with crystal violet.Six random fields of cells were counted in each well under a microscope at a magnification of $\times 100$. Three independent experiments were conducted.

\section{Wound-healing migration assay}

Cells were cultured in six-well plates (1.5×105 cells per well) and transfected with miRNA or siRNA. When the cells reached $90 \%$ confluence, a single wound was created in the center of the cell monolayer by using a T-200-Y pipet tip. Wound areas were visualized under an optical microscope with a magnification of $\times 100$. Cell migration capability was expressed by gap closure.

\section{Dual-luciferase reporter assay}

Luciferase reporter assay was performed as described previously [22]. The NPC cell line CNE$1\left(1.5 \times 10^{9}\right.$ cells per six-well plate) was co-transfected with miR-184 mimic or negative control; PGL3-Notch2 3'UTR wild-type vector or PGL3-Notch2 3'UTR mutant (mut) vector containing firefly luciferase reporter; and 3'UTR of the Notch2 gene (Promega, Madison, WI) using the transfection reagent Lipofectamine 2000 (Invitrogen, Carlsbad, CA). Cells were harvested $48 \mathrm{~h}$ after transfection. Luciferase activities were analyzed using the Dual-Luciferase Reporter Assay System (Promega) according to the manufacturer's protocol. Each experiment was done in triplicate.

\section{Western blot analysis}

After $48 \mathrm{~h}$ of transfection, cells were extracted and prepared in modified RIPA buffer (Beyotime, Shanghai, China). Total protein was extracted, and protein concentration was quantified using a BCA protein assay kit (Beyotime, Shanghai, China). A total of $20 \mathrm{mg}$ of protein from each sample was used for Western blot analysis.The primary antibodies used in this study included monoclonal anti-Notch2 (1:1000; Abcam, HK), anti-E-cadherin (1:1000; Cell Signaling Technology,USA), anti-N-cadherin (1:1000; Cell Signaling Technology,USA), anti-Vimentin (1:1000; Cell Signaling Technology,USA), and anti- $\beta$-actin (1:2000; Cell Signaling Technology, USA) was used as the loading control. Immunoreactive bands were visualized using ECL detection reagent (Millipore, Billerica, MA, USA). All data analyses were repeated three times independently.

\section{Tumour xenograft model}

A new experimental model of spontaneous lymph node metastasis was developed for the present study as previously described [23]. Athymic male BALB/c nude mice aged 6-8 weeks were obtained from the Medical Centre of Yangzhou University (Yangzhou, China). Spontaneous lymph node metastasis was then induced by injecting $2 \times 10^{6} \mathrm{CNE} 1$ cells into the footpad of individual nude mice. When the xenograft volumes reached $60 \mathrm{~mm}^{3}$, all the nude mice were divided into five groups ( $\mathrm{n}=5$ mice each) as follows: negative control group (CNE1/saline buffer) as the G1 group, control agomir group (CNE1/control agomir group) as the G2 group, miR-184 agomir group (CNE1/miR-184 agomir group) as the G3 group, control antagomir group (CNE1/control antagomir group) as the G4 group and miR-184 antagomir group (CNE1/miR-184 antagomir group) as the G5 group. Subsequently, $5 \mathrm{nmol}$ miR-184 agomir or control agomir (RiboBio) in $20 \mu \mathrm{l}$ of saline buffer was injected subcutaneously into the footpad to the tumour mass of each mouse in

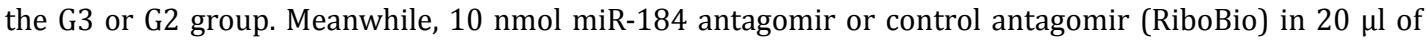
saline buffer was injected subcutaneously into the footpad to the tumour mass of each mouse in the G5 or G4 group. Finally, $20 \mu \mathrm{l}$ of saline buffer was injected subcutaneously into the footpad to the tumour mass of each mouse in the G1 group. All groups were injected twice a week, and the tumour volume (in $\mathrm{mm}^{3}$ ) 


\section{Cellular Physiology Cell Physiol Biochem 2018;49:1564-1576 \begin{tabular}{ll|l} 
and Biochemistry Published online: 13 September, 2018 & $\begin{array}{l}\text { (c) } 2018 \text { The Author(s). Published by S. Karger AG, Basel } \\
\text { www.karger.com/cpb }\end{array}$ \\
\hline
\end{tabular} \\ Zhu et al.: miR-184 Inhibits Tumor Invasion}

was measured by a calliper every 3-4 days and calculated using the modified ellipse formula (volume = length $\times$ width $^{2} / 2$ ). The experiment was terminated when the control primary tumour invaded the popliteal region or the mouse died. At 44 days after injection, the mice were killed, and tumours were weighed after necropsy. The presence of popliteal lymph node metastasis was observed by necropsy. Animal experiments were approved by the Animal Committee of Nanjing Origin Biosciences, China.

\section{Immunohistochemistry}

At the end of the study, all mice were sacrificed, and the tumors were surgically excised. Parts of the tumour tissues were fixed in $10 \%$ buffered formalin and embedded in paraffin. Hematoxylin and eosin staining was performed on the $6 \mu \mathrm{m}$-thick paraffin-embedded tumour sections for histological study. For immunohistochemistry, sections were incubated with primary antibodies against E-cadherin, $\mathrm{N}$-cadherin and vimentin (Cell Signalling Technology, USA) overnight at $4{ }^{\circ} \mathrm{C}$ after permeabilisation with a solution of $0.1 \%$ sodium citrate and $0.1 \%$ Triton X-100 and blocking with $10 \%$ rabbit serum. The slides were washed with PBS and then incubated with secondary antibody labelled with horseradish peroxidase at room temperature for $2 \mathrm{~h}$. After colour development by diaminobenzidine (Maixin Bio-Tech Co., Ltd), the slices were counterstained in hematoxylin and mounted with a neutral resin medium. The whole slide was first viewed at 100x magnification to identify a 'hot spot' representing the area of highest vessel density. The field was then switched to $400 \times$ magnification for analysis. For each slide, the epithelial-mesenchymal transition (EMT) was calculated as the average number of E-cadherin $(+), \mathrm{N}$-cadherin $(+)$ and $\operatorname{vimentin}(+)$ cells in eight visual fields.

\section{Statistical analysis}

Statistical analyses were performed through Student's t-test and one-way ANOVA with GraphPad Prism 7.0 software and SPSS 13.0. Data are expressed as the mean \pm standard deviation from at least three separate experiments. Differences were considered significant at $\mathrm{P}<0.05$.

\section{Results}

\section{MiR-184 is downregulated and Notch2 is upregulated in NPC cell lines}

To confirm the correlation between NPC tumourigenesis and miRNA gene expression, we performed quantitative reverse-transcription PCR (qRT-PCR) to determine the mRNA level of miR-184 in six NPC cell lines (5-8F, 6-10B, HNE1, SUNE1, CNE-1 and CNE-2) and NP69. MiR-184 expression levels were higher in NP69 than in the six NPC cell lines (Fig. 1A). By contrast, NP69 expressed the lowest amounts of the Notch2 mRNA and protein than the NPC cell lines (Fig. 1B and 1C). The results indicated that miR-184 was significantly downregulated and Notch2 is upregulated in NPC cell lines. Thus, miR-184 may be a tumor suppressor in NPC and may have a negative correlation with Notch2.

\section{MiR-184 overexpression inhibits invasion and migration in the NPC cell lines}

To examine whether miR-184 affects the migration and invasion of NPC cells, we performed cell wound-healing assay and Transwell experimental assays. MiR-184 mimic or NC was transfected into CNE-1 and CNE-2 cells. Following transfection, qRT-PCR was performed to detect miR-184 expression. qRT-PCR analysis results showed that the CNE-1 and CNE- 2 cells were transfected with miR-184 mimics, which significantly increased miR184 expression (Fig. 2A). Forcing miR-184 expression distinctly inhibited CNE-1 and CNE-2 cell migration and invasion relative to those in the control group (Fig. $2 \mathrm{~B}$ and $2 \mathrm{C}$ ). In the wound-healing assay, CNE-1 and CNE-2 cell migration was inhibited relative to that in the negative control group by miR-184 mimic transfection (Fig. 2D). MiR-184 overexpression obviously suppressed NPC cell migration and invasion, and these results suggest that miR184 expression exerted an anti-metastatic effect.

MiR-184 inhibition increases invasion and migration in NPC cells

To further determine the effect of miR-184 silencing on NPC's cell migration and invasive abilities, we also transiently transfected CNE-1 and CNE-2 cells with miR-184 inhibitor or a control and then performed wound-healing assays, Transwell migration assays and 
Fig. 1. MiR-184 was downregulated and Notch2 was upregulated in nasopharyngeal carcinoma cell lines. (A) Expression levels of miR-184 in six NPC cell lines and NP69 cells through qRT-PCR. $\mathrm{N}=3$ for each group. (B) Expression levels of Notch2 in six NPC cell lines and NP69 cells through qRTPCR. N=3 for each group. (C) Western blot analysis for Notch2 expression in six NPC cell lines and NP69 cells. $\beta$-actin antibody was used as the loading control.

invasion assays. MiR-184 inhibitor expression gradually decreased in the CNE-1 and CNE-2 cell lines (Fig. 2A). As expected, Transwell assay confirmed that the downregulated expression of miR-184 in CNE-1 and CNE-2 cells remarkably enhanced NPC cell migration and invasion relative to that in the control cells (Fig. 3A and 3B). Similarly, in the wound-healing assay, cell migration was significantly higher in the cells transfected with the miR-184 inhibitor than in the negative control group (Fig. 3C). These results indicate that miR184 silencing could promote NPC cell migration and invasion in vitro.

\section{MiR-184 inhibits EMT}

EMT is a key event in cancer invasion and metastasis; hence, we examined whether miR-184 can induce EMT in NPCs. We analysed the effect of miR-184 on EMT by detecting the protein levels of EMT markers. Results showed that miR-184 increased the expression of epithelial marker E-cadherin and decreased the expression of $\mathrm{N}$-cadherin and vimentin (Fig. 4). Meanwhile, after miR-184 inhibition, the protein E-cadherin level was reduced, whereas the $\mathrm{N}$-cadherin and vimentin levels were raised (Fig. 4). These findings indicate that miR-184 could suppress EMT.
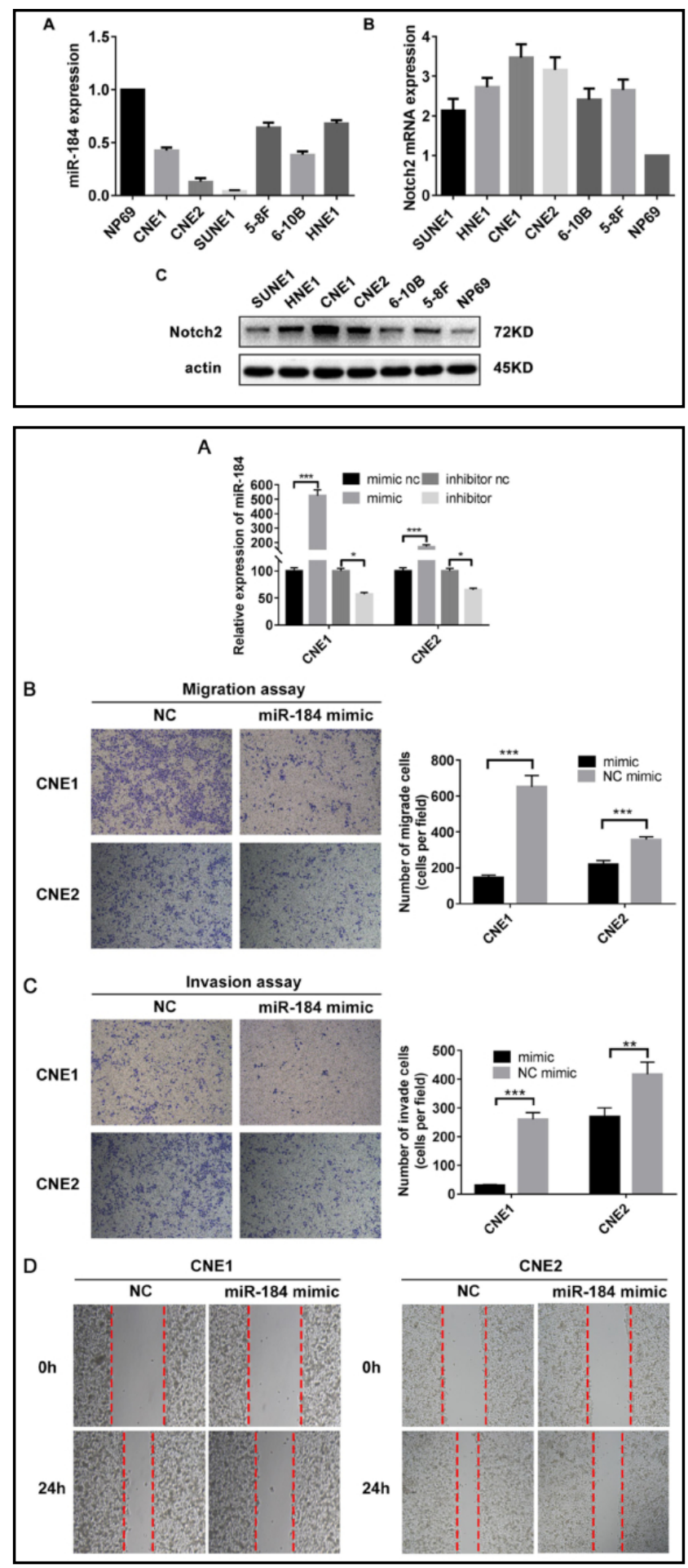

Fig. 2. Overexpression of MiR-184 decreases invasion and migration in CNE-1 and CNE-2 cell lines. (A) The relative expression levels of miR-184 after transfection. (B) The effects of miR-184 mimics on migration ability of NPC. (C) The invasion capacity of cells was investigated using a Matrigel invasion assay. (D) The migration of cells was examined using a wound healing assay. Results are presented as the mean \pm standard deviation $(\mathrm{n}=3) .{ }^{*} \mathrm{P}<0.05 ;{ }^{* *} \mathrm{P}<0.01 ;{ }^{* * *} \mathrm{P}<0.001$. 


\section{Cellular Physiology Cell Physiol Biochem 2018;49:1564-1576

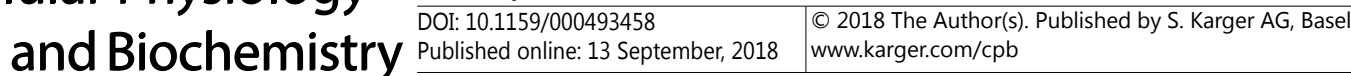

Notch2 is a direct target of miR-184 in NPC

To investigate the target gene of miR-184 in NPC carcinogenesis, we used three public databases (miRwalk, miRanda and Targetscan) to predict the potential target gene of miR-184. We found that Notch2 contained miR-184-binding sequences in its $3^{\prime}$-UTR regions (Fig. 5A). To verify whether miR-184 can repress the expression of Notch2 by directly interacting with the predicted binding site, we constructed luciferase reporter vectors that included wild-type (WT) and mutated Notch2 3'-UTR reporters. We then co-transfected these reporters with miR-184 mimic and mimic control into CNE1 cells. Luciferase assays showed that miR-184 significantly inhibited luciferase activity of the WT Notch2-3'-UTR reporter gene, whereas the activity of the mutant reporter gene was unaffected (Fig. 5B). To further confirm that Notch2 acts as a miR-184 target, we analysed the Notch2 mRNA and protein expression levels in the CNE- 1 and CNE-2 cells transfected with miR-184 mimic or inhibitor by qRT-PCR and Western blot. Notch2 expression simultaneously decreased at the mRNA and protein levels after miR-184 overexpression (Fig. 5C and 5D). Reciprocally, miR-184 knockdown was accompanied with augmented Notch2 expression in the CNE1 cells (Fig. $5 \mathrm{C}$ and $5 \mathrm{E}$ ). These results indicate that Notch2 was directly and negatively regulated by miR-184.

Notch2 promotes EMT in NPC Notch2 has been found to be involved in cell migration and invasion. A previous study showed that Notch2 knockdown can suppresses gliomal cell invasion [24]. Recent investigations have indicated that Notch2 promotes EMT in oesophageal squamous cell cancer [25]. To further prove whether Notch2 is associated with NPC cell migration and invasion, we transiently transfected CNE-1 and CNE-2 cells with Notch2-small interference RNA (siRNA) or NC-siRNA and conducted wound-healing assays, Transwell assays and Western blot to examine the effect of Notch2 on

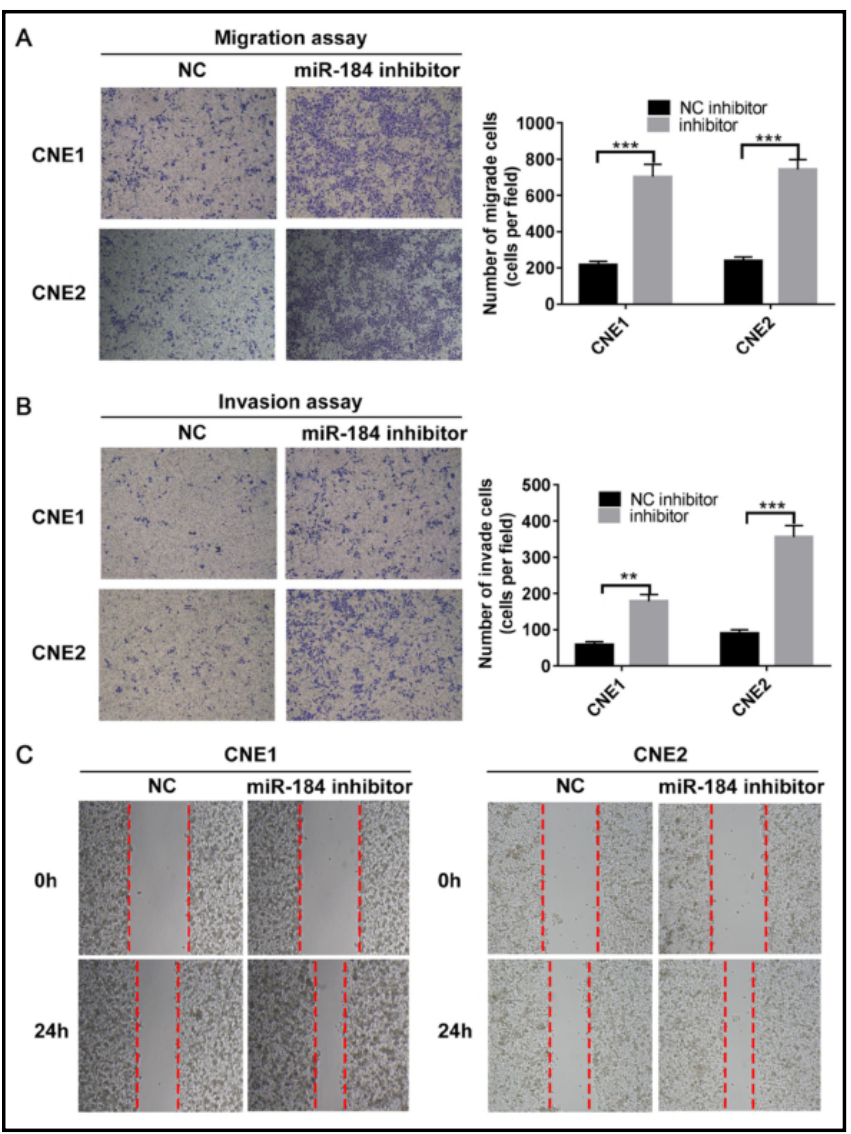

Fig. 3. Inhibition of MiR-184 increases invasion and migration in CNE-1 and CNE-2 cell lines. (A) The effects of miR-184 inhibitor on migration ability of NPC. (B) The invasion capacity of cells was investigated using a Matrigel invasion assay. (C) The migration of cells was examined using a wound healing assay. Results are presented as the mean \pm standard deviation $(n=3)$. ${ }^{* *} \mathrm{P}<0.01 ;{ }^{* * *} \mathrm{P}<0.001$.

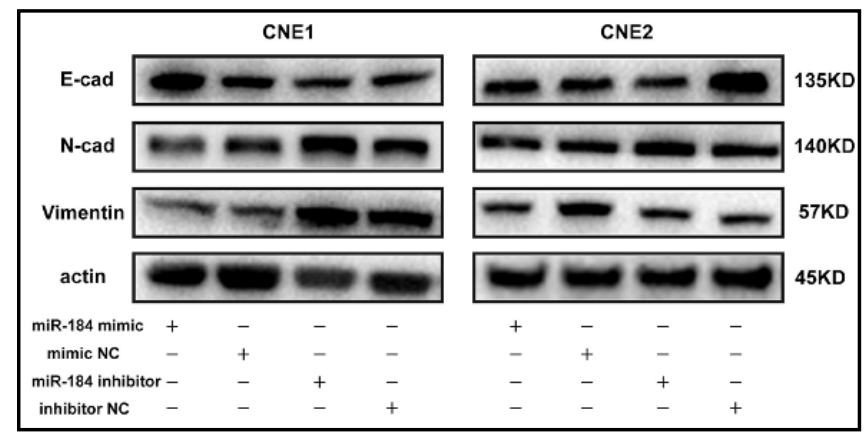

Fig. 4. miR-184 mimics and inhibitor led to the changes of EMT markers'expression. 


\section{Cellular Physiology Cell Physiol Biochem 2018;49:1564-1576

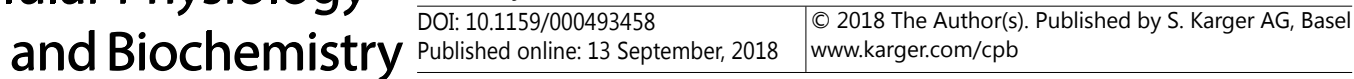 \\ Zhu et al.: miR-184 Inhibits Tumor Invasion}

the EMT process. After transfection, the mRNA expression of Notch2 obviously decreased as confirmed by qRTPCR (Fig. 6A). The downregulation of Notch2 significantly decreased the migration and invasion ability of CNE-1 and CNE-2 cells (Fig. 6B and $6 \mathrm{C}$ ). As expected, Notch2 silencing inhibited the woundclosing activities of the CNE-1 and CNE- 2 cells compared with those in the controls (Fig. 6D). Western blot revealed that the expression levels of $\mathrm{N}$-cadherin and vimentin were diminished, whereas that of E-cadherin was raised, in the anti-Notch2 group (Fig. 6E).

MiR-184 inhibits EMT by suppressing Notch2

To evaluate the molecular mechanism by which miR-184 inhibits invasion and metastasis in NPC cells, we assembled four groups for analysis. These groups were miR-184-NC with Notch2-NC, anti-miR-184 with si-Notch2, anti-miR-184 with Notch2-NC and miR-184-NC with si-Notch2. The miR-184 inhibitor increased the invasion and migration abilities of NPC cells, whereas shRNA-Notch2 abolished this change (Fig. 7A and 7B). We then examined the expression of the epithelial marker E-cadherin and mesenchymal markers vimentin and N-cadherin by Western blot. The miR-184 inhibitor reduced the EMT-related protein E-cadherin levels and increased the N-cadherin and vimentin levels in the CNE-1 and CNE-2 cells. However, co-transfection with shRNA-Notch2 partially abolished these alterations at the protein level (Fig. 7C). These results suggest that miR-184 inhibited EMT by suppressing Notch2 expression.

\section{MiR-184 inhibits NPC metastasis by decreasing EMT in vivo}

To further investigate the effect of miR-184 on NPC metastasis in vivo, CNE- 1 cell $\left(2 \times 10^{6}\right.$ cells) were injected into the footpad of nude mice. When the xenograft volumes reached 60 $\mathrm{mm}^{3}$, corresponding reagents were subcutaneously injected into the footpad to the tumour mass of every mouse in each group. Upon autopsy after 44 days, the tumour was removed and weighed, and tumour popliteal lymph node metastasis progression was observed. Results showed that miR-184 overexpression significantly reduced the primary tumour size relative to that in the agomir-NC and negative control, and miR-184 inhibition evidently increased the tumour sizes relative to those in the antagomir-NC and negative control (Fig. 8A and 8B). Similarly, the tumour weight and mean volume demonstrated the same results with size (Fig. 8C and 8D). We then studied the effect of miR-184 on tumour metastasis in vivo. Exogenous miR-184 suppressed the cell invasion and migration of NPC cells in vitro; thus, we reasoned that miR-184 may also inhibit metastasis in vivo. Indeed, miR-184 obviously inhibited tumour popliteal lymph node metastasis (Fig. 8A, 8B and 8E). Immunohistochemistry assays also showed that endogenous miR-184 caused similar changes in the EMT markers in vivo (Fig. 9A, B, C, D and E). Collectively, the data suggest that miR-184 inhibited distal metastases in vivo.

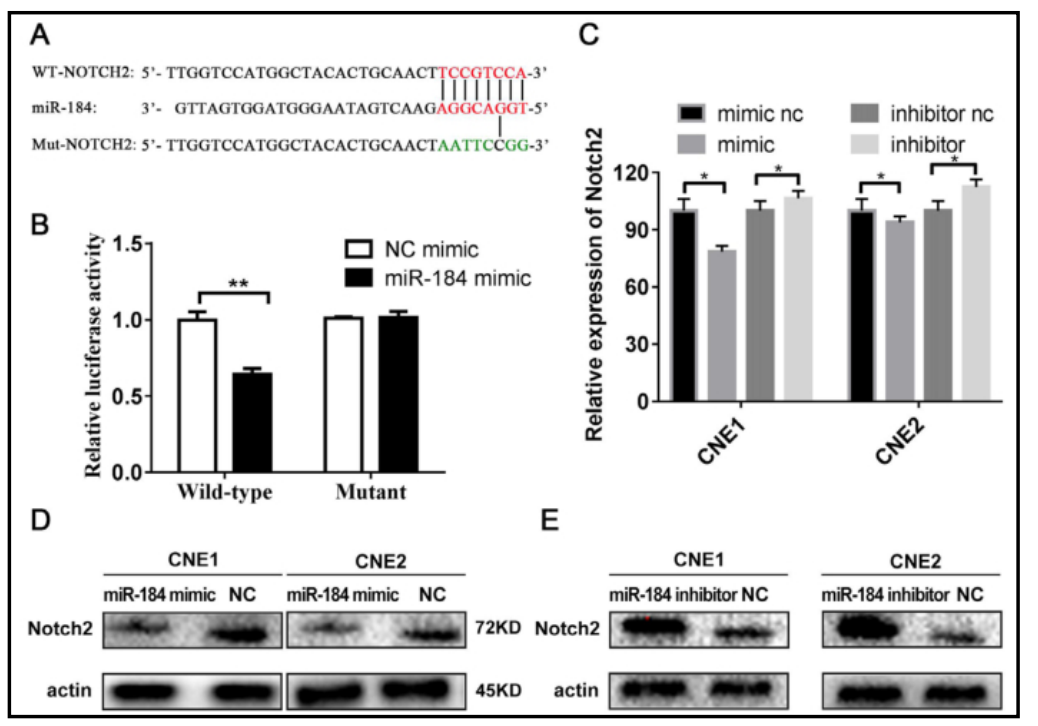

Fig. 5. The prediction and validation of miR-184 target gene. (A)The
prediction and validation of miR-184 target gene. (B) Luciferase assays validated that Notch2 is a target of miR-184. (C,D and E) Further evidence of miR-184 targeting Notch2 by qRT-PCR, and western blotting. 


\section{Discussion}

In this study, we initially compared the significantly decreased expression of miR-184 in NPC cells against that in the immortalised nasopharyngeal epithelial cell line NP69 by qRT-PCR. The functions of miR184 in NPC cell invasion and metastasis were also analysed by transfecting miR-184 mimic and inhibitor into CNE-1 and CNE-2 NPC cells. MiR-184 significantly inhibited the migration and invasion of NPC cells in vitro. In this work, we also found that miR184 inhibited NPC cell invasion and metastasis by directly targeting Notch2, which regulates EMT progression. Overexpressing miR184 suppressed NPC metastasis in an established experimental xenograft model. Through a series of in vitro and in vivo assays, we uncovered that miR-184 served a vital role in regulating NPC invasion and metastasis.

A c c u m u lat in g evidence has shown that miR-184 expression is dysregulated in human cancers types. Such expression was found to be downregulated in glioma, and ectopic miR-184 expression suppressed the proliferation and invasion of gliomal cells by directly regulating SND1 [14]. In human colorectal cancer, miR-184 was downregulated in CRC tissues and cell lines, which significantly inhibited proliferation, migration and invasion in human colorectal cancer by directly targeting IGF-1R [26]. However, some studies have suggested miR-184 as a potential onco-miR in various human tumours. Dysregulated miR184 has been reported as a potential oncomir, which contributes to cell proliferation and cell apoptosis inhibition in tongue carcinoma [20]. Moreover, previous studies indicated that miRNA-184 acts as an oncogenic regulator by targeting INPPL1 in HCC development [27]. We

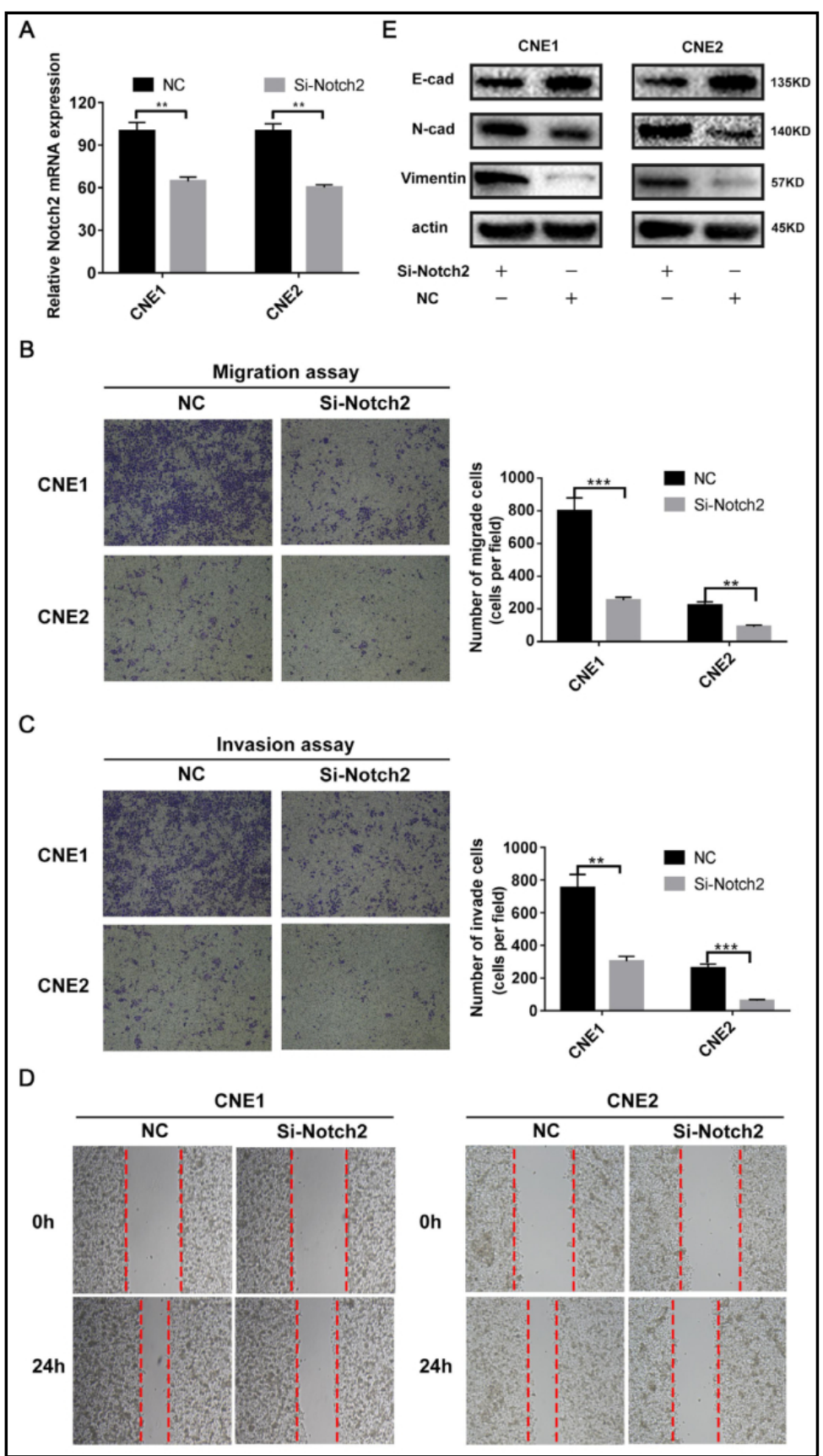

Fig. 6. Notch2 promotes EMT of NPC. (A) shRNA transfection of Notch2 could inhibit its expression. (B, D) shRNA-Notch2 inhibited migration ability of NPC. (C) shRNA-Notch2 inhibited invasion ability of NPC. (E) shRNA-Notch2 regulated EMT markers of NPC. ${ }^{* *} \mathrm{P}<0.01 ;{ }^{* * *} \mathrm{P}<0.001$. 
Fig. 7. The miR184-mediated EMT process depends on Notch2. (A, B) miR184 inhibitor caused an increase in NPC cells'migration and whereas shRNANotch2 could abolish this change. (C) miR-184 inhibitor led to increase of N-cadherin and Vimentin and decrease of E-cadherin, whereas co-transfection with shRNA-Notch2 partially abolished these changes. ${ }^{*} \mathrm{P}<0.05$; ${ }^{* *} \mathrm{P}<0.01$. invasive ability,

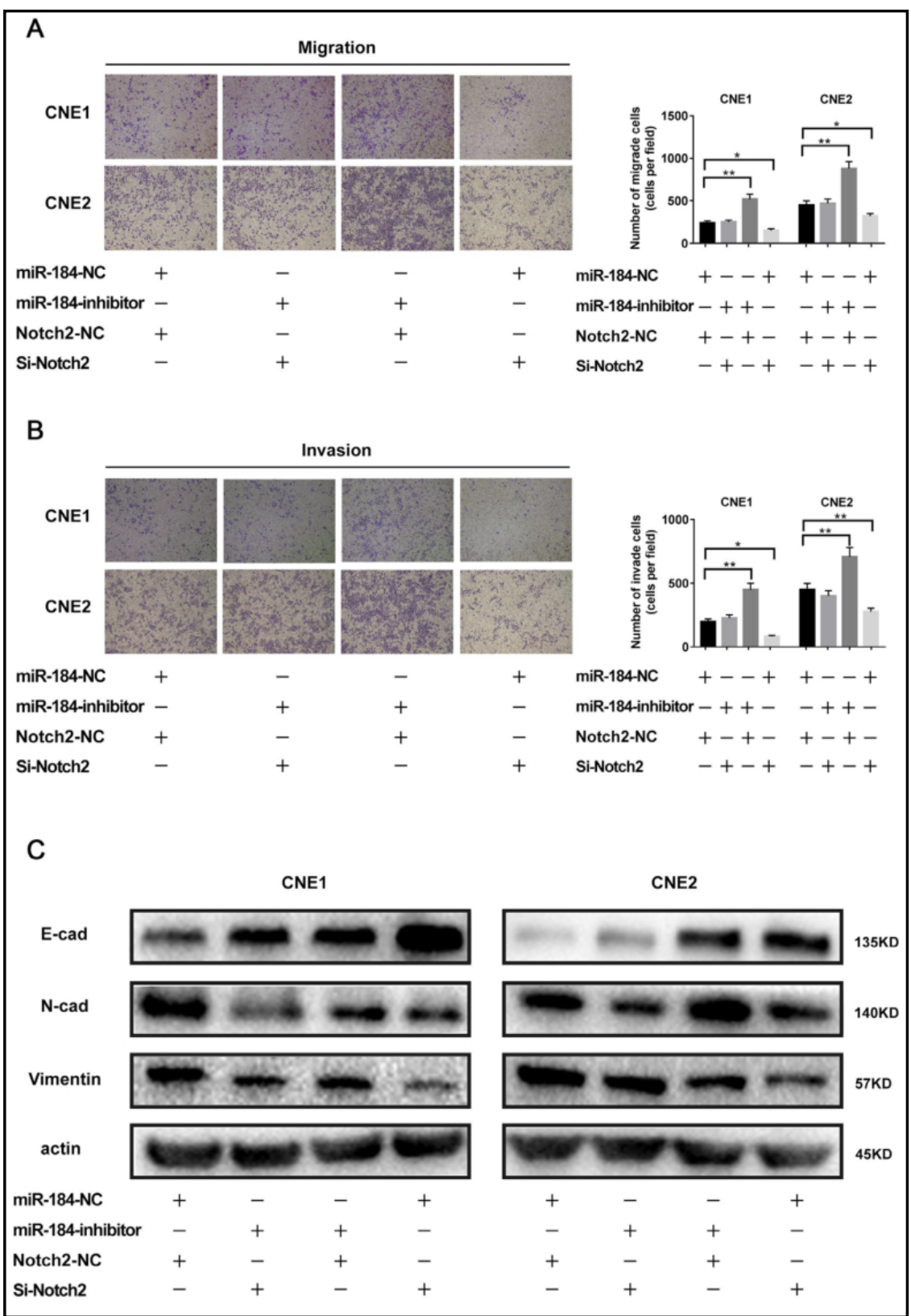

observed a difference between one normal sample and 6 NPC cell lines suggesting that miR184 could be downregulated in NPC cell lines. Upregulation and downregulation without other indications could be interpreted as differences between two populations. Collectively, these studies demonstrated the possible role of miR-184 in regulating cancer carcinogenesis and progression.

In previous studies, miR-184, identified as a potential tumour suppressor, inhibited cell growth by modulating the C-MYC-controlled cell cycle transition and BCL2-repressed mitochondrial apoptosis in NPC[21]. In our work, we further investigated the mechanisms of miR-184 in NPC invasion and metastasis. Results showed that miR-184 inhibited NPC invasion and metastasis through EMT inhibition by suppressing Notch2 expression. Similarly, Notch2 knockdown exerted similar effects to those of miR-184 overexpression in NPC cells.

EMT is a critical process in promoting stationary tumour cells to migrate and invade. Such a phenomenon is a developmental program that enables stationary epithelial cells to gain migratory and invasive abilities as single cells [28]. This progression can be triggered 


\section{Cellular Physiology Cell Physiol Biochem 2018;49:1564-1576 \begin{tabular}{l|l|l} 
DOI: 10.1159/000493458 & O 2018 The Author(s). Published by S. Karger AG, Basel \\
www.karger.com/cpb
\end{tabular} \\ Zhu et al.: miR-184 Inhibits Tumor Invasion}

Fig. 8. miR-184 inhibited NPC metastasis via decrease EMT in vivo. $(A, B)$ Photographs of mice injected with agomir-NC or agomir-184, antagomir-NC or antagomir-184, negative control.The metastasis nodules are indicated by arrows.

Tumor weight averages between negative control, agomirNC, agomir-184, antagomirNC and antagomir-184 mice groups at the end of the experiment (day 41). (D) Graph representing tumor volumes averages between negative control, agomir-NC, agomir-184, antagomir-NC and antagomir-184 mice groups at the end of the experiment (day 41). five mice in each group. (E) The graph gives the incidences of metastasis in mice that had received footpad injections of each cell line. ${ }^{*} \mathrm{P}<0.05$; ${ }^{* *} \mathrm{P}<0.01,{ }^{* * *} \mathrm{P}<0.001$.

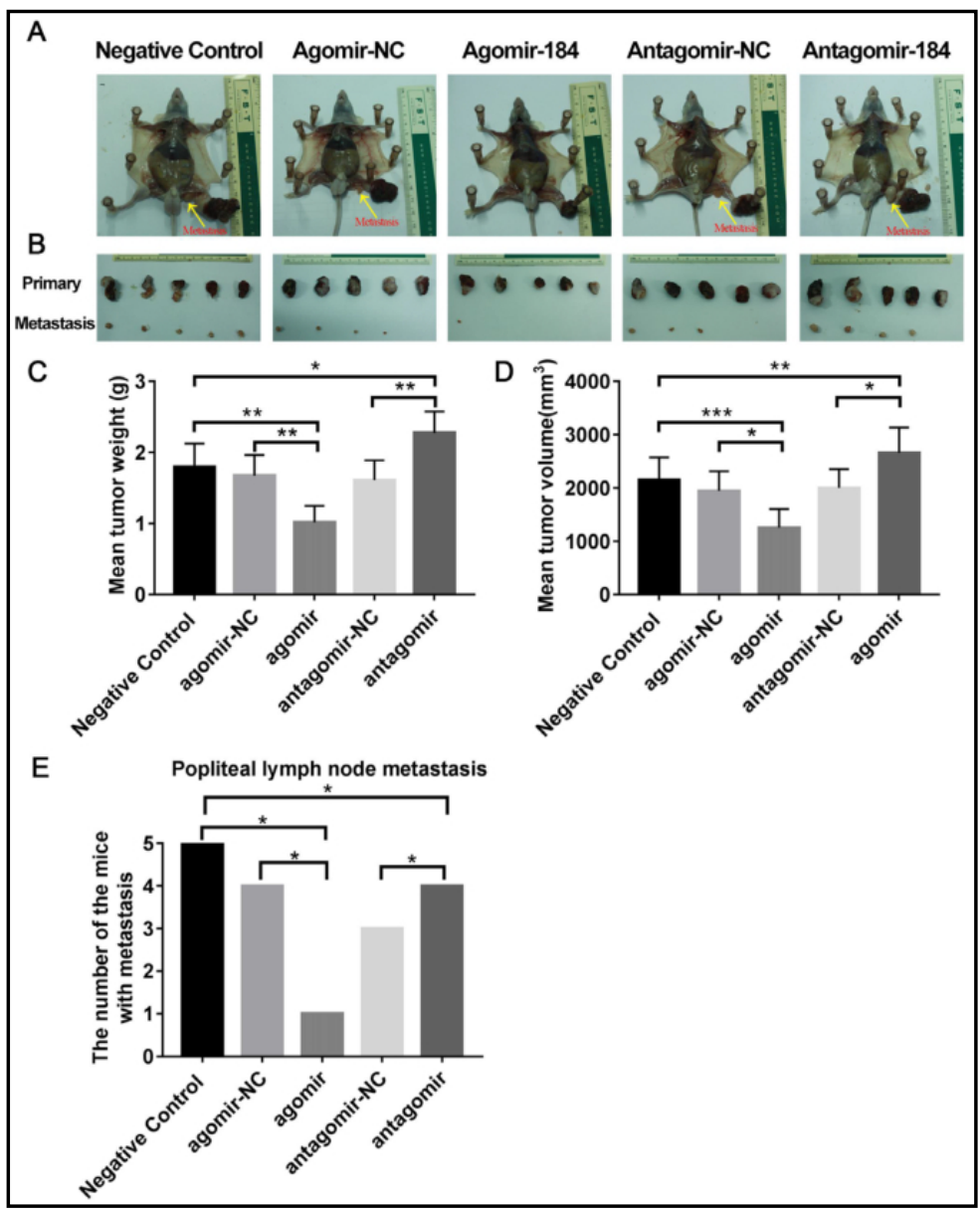

by various developmental signalling pathways, such as tumour growth factor beta, Wnt, Notch, growth factor receptor tyrosine kinases and PLC- $\gamma$ pathways [25]. Notch signalling is an evolutionarily conserved pathway involved in EMT and metastasis [29-33]. In mammals, Notch signalling includes four transmembrane Notch receptors (Notch-1, Notch-2, Notch-3 and Notch-4) and five canonical transmembrane ligands (Delta-like DLL1,DLL3, DLL4, Jagged-1 and Jagged-2)[34]. Amongst these molecules, Notch2 is associated with proliferation, invasion and metastasis in numerous tumour types, such as gastric carcinoma $[24,35]$, laryngeal squamous cell carcinoma [36] and salivary adenoid cystic carcinoma [37]. In the present study, we elucidated the molecular mechanism by which miR-184 inhibits NPC cell invasion and metastasis by directly targeting Notch2. To achieve this goal, we examined the effect of Notch2 on the EMT process by wound-healing assay, Transwell assay and Western blot. Notch2 knockdown decreased EMT progression and CNE-1 and CNE-2 cell invasion. Notably, the inhibitory effects of miR-184 on EMT progression and cell invasion were partially abrogated by Notch 2 knockdown in NPC cells. Thus, these data clearly demonstrated that miR-184 inhibited NPC invasion and metastasis by repressing EMT progression through Notch2 suppression. 


\section{Cellular Physiology Cell Physiol Biochem 2018;49:1564-1576

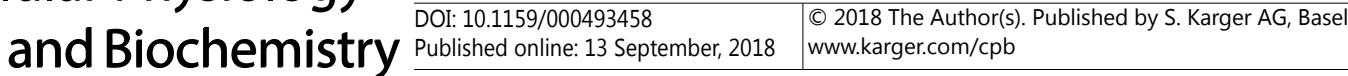 \\ Zhu et al.: miR-184 Inhibits Tumor Invasion}

Fig. 9. (A) HE staining result of the tumor formed in nude mice using, paraffinembedded tumor sections were stained with antivimentin, E-cadherin and $\mathrm{N}$-cadherin. Representative figures were shown. (B,C and D) Primary tumor E-cadherin, $\mathrm{N}$-cadherin, Vimentin expression levels in $\mathrm{HE}$ staining. (E) In agomir-184 group, primary tumor and metastasis tumor E-cadherin, $\mathrm{N}$-cadherin, Vimentin expression levels in $\mathrm{HE}$ staining. ${ }^{*} \mathrm{P}<0.05$; ${ }^{* *} \mathrm{P}<0.01$; $* * * \mathrm{P}<0.001$.

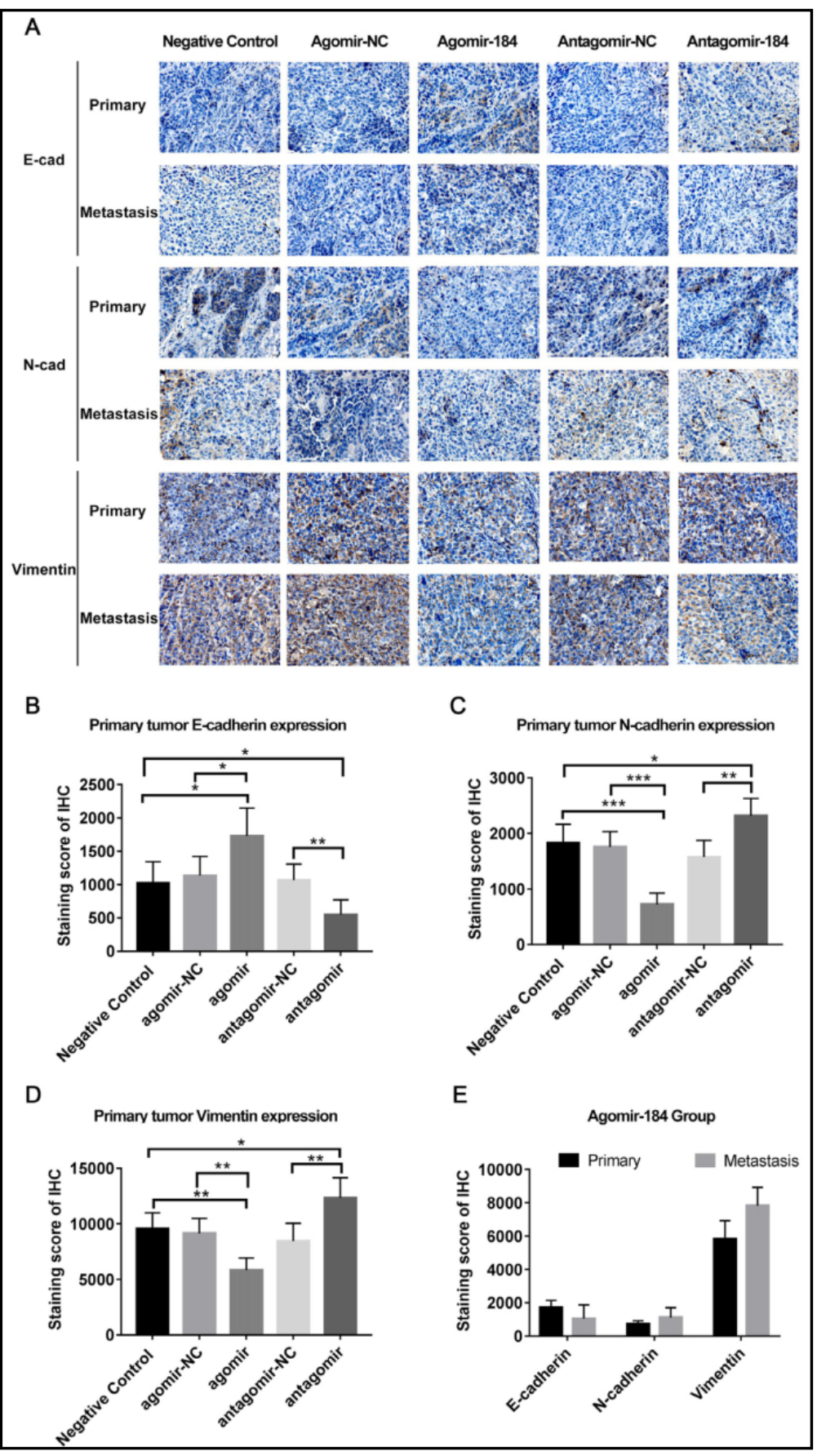

\section{Conclusion}

Our results demonstrated that miR-184 expression was downregulated in NPC cell lines. MiR-184 also evidently suppressed NPC invasion and metastasis in vitro and in vivo. Mechanistically, miR-184 inhibited the EMT progression of NPC by repressing Notch2. Collectively, we believe that miR-184 plays an essential role in NPC progression and may serve as a promising therapeutic target for NPC treatment in the future. 


\section{Cellular Physiology Cell Physiol Biochem 2018;49:1564-1576 and Biochemistry Published \begin{tabular}{l|l} 
DOI: 10.1159/000493458 & $\begin{array}{l}\text { (c) } 2018 \text { The Author(s). Published by S. Karger AG, Basel } \\
\text { www.karger.com/cpb }\end{array}$
\end{tabular}}

Zhu et al.: miR-184 Inhibits Tumor Invasion

\section{Acknowledgements}

We thank the members of the Research Center of Clinical Oncology of of Jiangsu province for their technical assistance. This study was supported by the National Natural Science Foundation of China (81672989), the Jiangsu Provincial Commission of Health and Family Planning Youth Fund (Q201501), the JiangSu Clinical Medicine Science and Technology Special Fund (No. BL2014091), the Young Medical Talent Fund of JiangSu Provincial Department for Health (QNRC2016648).

\section{Disclosure Statement}

The authors declare that they have no competing interests.

\section{References}

$>1$ Wang JG, Tang WP, Liao MC, Liu YP, Ai XH: miR-99a suppresses cell invasion and metastasis in nasopharyngeal carcinoma through targeting HOXA1. Onco Targets Ther 2017;10:753-761.

2 Yang W, Lan X, Li D, Li T, Lu S: MiR-223 targeting MAFB suppresses proliferation and migration of nasopharyngeal carcinoma cells. BMC Cancer 2015;15:461.

3 Jiang C, Wang H, Zhou L, Jiang T, Xu Y, Xia L: MicroRNA-212 inhibits the metastasis of nasopharyngeal carcinoma by targeting SOX4. Oncol Rep 2017;38:82-88.

4 Sin TK, Wang F, Meng F, Wong SC, Cho WC, Siu PM, Chan LW, Yung BY: Implications of MicroRNAs in the Treatment of Gefitinib-Resistant Non-Small Cell Lung Cancer. Int J Mol Sci 2016;17:237.

5 Aleckovic M, Kang Y: Regulation of cancer metastasis by cell-free miRNAs. Biochim Biophys Acta 2015;1855:24-42.

6 Tan G, Tang X, Tang F: The role of microRNAs in nasopharyngeal carcinoma. Tumour Biol 2015;36:69-79.

7 Mao Y, Wu S, Zhao R, Deng Q: MiR-205 promotes proliferation, migration and invasion of nasopharyngeal carcinoma cells by activation of AKT signalling. J Int Med Res 2016;44:231-240.

-8 Li YQ, He QM, Ren XY, Tang XR, Xu YF, Wen X, Yang XJ, Ma J, Liu N: MiR-145 inhibits metastasis by targeting fascin actin-bundling protein 1 in nasopharyngeal carcinoma. PLoS One 2015;10:e0122228.

-9 Wang F, Jiang C, Sun Q, Yan F, Wang L, Fu Z, Liu T, Hu F: Downregulation of miR429 and inhibition of cell migration and invasion in nasopharyngeal carcinoma. Mol Med Rep 2016;13:3236-3242.

10 Chen JH, Yang R, Zhang W, Wang YP: Functions of microRNA-143 in the apoptosis, invasion and migration of nasopharyngeal carcinoma. Exp Ther Med 2016;12:3749-3755.

11 Wu M, Ye X, Wang S, Li Q, Lai Y, Yi Y: MicroRNA-148b suppresses proliferation, migration, and invasion of nasopharyngeal carcinoma cells by targeting metastasis-associated gene 2 . Onco Targets Ther 2017;10:2815-2822.

12 Jiang C, Qin B, Liu G, Sun X, Shi H, Ding S, Liu Y, Zhu M, Chen X, Zhao C: MicroRNA-184 promotes differentiation of the retinal pigment epithelium by targeting the AKT2/mTOR signaling pathway. Oncotarget 2016;7:52340-52353.

13 Zhi F, Shao N, Xue L, Xu Y, Kang X, Yang Y, Xia Y: Characteristic MicroRNA Expression Induced by deltaOpioid Receptor Activation in the Rat Liver Under Prolonged Hypoxia. Cell Physiol Biochem 2017;44:22962309.

14 Emdad L, Janjic A, Alzubi MA, Hu B, Santhekadur PK, Menezes ME, Shen XN, Das SK, Sarkar D, Fisher PB: Suppression of miR-184 in malignant gliomas upregulates SND1 and promotes tumor aggressiveness. Neuro Oncol 2015;17:419-429.

15 Cheng Z, Wang HZ, Li X, Wu Z, Han Y, Li Y, Chen G, Xie X, Huang Y, Du Z, Zhou Y: MicroRNA-184 inhibits cell proliferation and invasion, and specifically targets TNFAIP2 in Glioma. J Exp Clin Cancer Res 2015;34:27.

16 Liang XG, Meng WT, Hu LJ, Li L, Xing H, Xie G, Wang AQ, Jia YQ: MicroRNA-184 Modulates Human Central Nervous System Lymphoma Cells Growth and Invasion by Targeting iASPP. J Cell Biochem 2017;118:26452653.

17 Su Z, Chen D, Li Y, Zhang E, Yu Z, Chen T, Jiang Z, Ni L, Yang S, Gui Y, Ye J, Lai Y: microRNA-184 functions as tumor suppressor in renal cell carcinoma. Exp Ther Med 2015;9:961-966. 


\section{Cellular Physiology Cell Physiol Biochem 2018;49:1564-1576 \begin{tabular}{l|l|l} 
and Biochemistry & DOI: 10.1159/000493458 & $\begin{array}{l}\text { C } 2018 \text { The Author(s). Published by S. Karger AG, Basel } \\
\text { www.karger.com/cpb }\end{array}$
\end{tabular}

18 Li H, Xiang H, Ge W, Wang H, Wang T, Xiong M: Expression and functional perspectives of miR-184 in pancreatic ductal adenocarcinoma. Int J Clin Exp Pathol 2015;8:12313-12318.

19 Wu GG, Li WH, He WG, Jiang N, Zhang GX, Chen W, Yang HF, Liu QL, Huang YN, Zhang L, Zhang T, Zeng XC: Mir-184 post-transcriptionally regulates SOX7 expression and promotes cell proliferation in human hepatocellular carcinoma. PLoS One 2014;9:e88796.

20 Wong TS, Liu XB, Wong BY, Ng RW, Yuen AP, Wei WI: Mature miR-184 as Potential Oncogenic microRNA of Squamous Cell Carcinoma of Tongue. Clin Cancer Res 2008;14:2588-2592.

21 Zhen Y, Liu Z, Yang H, Yu X, Wu Q, Hua S, Long X, Jiang Q, Song Y, Cheng C, Wang H, Zhao M, Fu Q, Lyu X, Chen Y, Fan Y, Liu Y, Li X, Fang W: Tumor suppressor PDCD4 modulates miR-184-mediated direct suppression of C-MYC and BCL2 blocking cell growth and survival in nasopharyngeal carcinoma. Cell Death Dis 2013;4:e872.

-22 Wu J, Yin L, Jiang N, Guo WJ, Gu JJ, Chen M, Xia YY, Wu JZ, Chen D, Wu JF, Wang DJ, Zong D, Zhang N, Ding K, Huang T, He X: MiR-145, a microRNA targeting ADAM17, inhibits the invasion and migration of nasopharyngeal carcinoma cells. Exp Cell Res 2015;338:232-238.

23 Hayashi K, Zhao M, Yamauchi K, Yamamoto N, Tsuchiya H, Tomita K, Hoffman RM: Cancer metastasis directly eradicated by targeted therapy with a modified Salmonella typhimurium. J Cell Biochem 2009;106:992-998.

24 Chen L, Chen XR, Zhang R, Li P, Liu Y, Yan K, Jiang XD: MicroRNA-107 inhibits glioma cell migration and invasion by modulating Notch2 expression. J Neurooncol 2013;112:59-66.

25 Wang C, Zhang W, Zhang L, Chen X, Liu F, Zhang J, Guan S, Sun Y, Chen P, Wang D, Un Nesa E, Cheng Y, Yousef GM: miR-146a-5p mediates epithelial-mesenchymal transition of oesophageal squamous cell carcinoma via targeting Notch2. Br J Cancer 2016;115:1548-1554.

-26 Wu G, Liu J, Wu Z, Wu X, Yao X: MicroRNA-184 inhibits cell proliferation and metastasis in human colorectal cancer by directly targeting IGF-1R. Oncol Lett 2017;14:3215-3222.

27 Gao B, Gao K, Li L, Huang Z, Lin L: miR-184 functions as an oncogenic regulator in hepatocellular carcinoma (HCC). Biomed Pharmacother 2014;68:143-148.

-28 Yeung KT, Yang J: Epithelial-mesenchymal transition in tumor metastasis. Mol Oncol 2017;11:28-39.

29 Wang Z, Li Y, Kong D, Sarkar FH: The role of Notch signaling pathway in epithelial-mesenchymal transition (EMT) during development and tumor aggressiveness. Curr Drug Targets 2010;11:745-751.

30 Shao S, Zhao X, Zhang X, Luo M, Zuo X, Huang S, Wang Y, Gu S, Zhao X: Notch1 signaling regulates the epithelial-mesenchymal transition and invasion of breast cancer in a Slug-dependent manner. Mol Cancer 2015;14:28.

-31 Zhao ZL, Ma SR, Wang WM, Huang CF, Yu GT, Wu TF, Bu LL, Wang YF, Zhao YF, Zhang WF, Sun ZJ: Notch signaling induces epithelial-mesenchymal transition to promote invasion and metastasis in adenoid cystic carcinoma. Am J Transl Res 2015;7:162-174.

32 Zhang L, Sha J, Yang G, Huang X, Bo J, Huang Y: Activation of Notch pathway is linked with epithelialmesenchymal transition in prostate cancer cells. Cell Cycle 2017;16:999-1007.

-33 Yang J, Guo W, Wang L, Yu L, Mei H, Fang S, Chen A, Liu Y, Xia K, Liu G: Notch signaling is important for epithelial-mesenchymal transition induced by low concentrations of doxorubicin in osteosarcoma cell lines. Oncol Lett 2017;13:2260-2268.

-34 Espinoza I, Pochampally R, Xing F, Watabe K, Miele L: Notch signaling: targeting cancer stem cells and epithelial-to-mesenchymal transition. Onco Targets Ther 2013;6:1249-1259.

-35 Tseng YC, Tsai YH, Tseng MJ, Hsu KW, Yang MC, Huang KH, Li AF, Chi CW, Hsieh RH, Ku HH, Yeh TS: Notch2induced COX-2 expression enhancing gastric cancer progression. Mol Carcinog 2012;51:939-951.

-36 Zou Y, Fang F, Ding YJ, Dai MY, Yi X, Chen C, Tao ZZ, Chen SM: Notch 2 signaling contributes to cell growth, anti-apoptosis and metastasis in laryngeal squamous cell carcinoma. Mol Med Rep 2016;14:3517-3524.

37 Qu J, Song M, Xie J, Huang XY, Hu XM, Gan RH, Zhao Y, Lin LS, Chen J, Lin X, Zheng DL, Lu YG: Notch2 signaling contributes to cell growth, invasion, and migration in salivary adenoid cystic carcinoma. Mol Cell Biochem 2016;411:135-141. 